\title{
Efeito da LUZ E dA Temperatura NA GeRminaçÃo de Alternathera tenella, Conyza bonariensis E Digitaria ciliaris ${ }^{1}$
}

\author{
Effect of Light and Temperature on Alternathera tenella, Conyza bonariensis and \\ Digitaria ciliaris Germination
}

VIVIAN, R. ${ }^{2}$, GOMES JR., F.G. ${ }^{2}$, CHAMMA, H.M.C.P. ${ }^{3}$, SILVA, A.A. ${ }^{4}$, FAGAN, E.B. ${ }^{5}$ e RUIZ, S.T. ${ }^{6}$

\begin{abstract}
RESUMO - Este trabalho teve o objetivo de avaliar a influência da luz e da temperatura na germinação de três espécies de plantas daninhas comuns em lavouras brasileiras. As espécies estudadas foram Alternanthera tenella, Conyza bonariensis e Digitaria ciliaris, nas quais se testou a influência de quatro variações de temperatura (15/25, 20/30, 20/35 e 30 / $40{ }^{\circ} \mathrm{C}$ ), com e sem a presença de luz, no percentual de germinação e no indice de velocidade de germinação (IVG). As sementes foram coletadas em canteiros experimentais, sendo elas selecionadas para permitir a homogeneização amostral. Posteriormente, 50 sementes de cada espécie foram colocadas em caixas do tipo gerbox, previamente preparadas com papel duplo umedecido de germinação "germitest". As caixas foram armazenadas em germinadores verticais tipo BOD, com controle de temperatura. As temperaturas foram mantidas na proporção de 16/8 horas diurna e noturna, respectivamente. As avaliações iniciaram-se aos três dias após incubação, sendo consideradas germinadas as sementes com protusão radicular igual ou superior a $1 \mathrm{~mm}$. Constatou-se efeito significativo da luz para a germinação de todas as espécies, principalmente para $D$. ciliaris. A. tenella apresentou germinação elevada em todas as variações de temperatura testadas, embora $30 / 40{ }^{\circ} \mathrm{C}$ tenham restringido a germinação de C. bonariensis. Os tratamentos também afetaram o IVG, cujo comportamento foi similar ao percentual de germinação, sendo o maior efeito constatado em $D$. ciliaris.
\end{abstract}

Palavras-chave: sementes fotoblásticas, velocidade de germinação, plantas daninhas.

\begin{abstract}
The objective of this work was to evaluate the effect of light and temperature on the germination of three common Brazilian weed species: Alternathera tenella, Conyza bonariensis and Digitaria ciliaris. The influence of fourtemperature variations (15/25, 20/30, 20/35 and $\left.30 / 40{ }^{\circ} \mathrm{C}\right)$ with and without light was tested on germination percentage and germination velocity rate (GVR). The seeds were collected in experimental seedbeds to allow sample homogenization. Fifty seeds of each species were placed in gerbox type boxes, previously prepared with humidified "germitest" germination double layer paper. The boxes were stored in temperature-controlled BOD type vertical germinators. The temperatures were maintained at the ratio of $16 / 8$ hours day and night, respectively. Evaluations started three days after incubation, with the seeds displaying roots equal or over $1 \mathrm{~mm}$ being considered to have germinated. A significant light effect on germination was confirmed fort all the species, mainly $\boldsymbol{D}$. ciliaris. A. tenella, presented high germination at all temperatures tested, even though $30 / 40{ }^{\circ} \mathrm{C}$ restricted $\mathbf{C}$. bonariensis germination. The treatments also affected GVR, whose behavior was similar to the germination percentage, with the highest effect being confirmed in $\boldsymbol{D}$. ciliaris.
\end{abstract}

Keywords: photoblastic seeds, germination velocity, weeds.

1 Recebido para publicação em 10.8.2007 e na forma revisada em 12.3.2008.

2 Doutorando do Dep. de Fitotecnia - DFT, Escola Superior de Agricultura Luiz de Queiroz - ESALQ/USP, 13416-401 PiracicabaSP, <agrovivian@yahoo.com.br>. ${ }^{3}$ Responsável técnico pelo Lab. Sementes - ESALQ/USP. ${ }^{4}$ Professor do Dep. de Fitotecnia DFT, Universidade Federal de Viçosa - UFV, 36570-000 Viçosa-MG. ${ }^{5}$ Professor do Dep. de Fisiologia Vegetal - Universidade de Patos de Minas - UNIPAM - Patos de Minas, MG. ${ }^{6}$ Mestranda do Dep. de Fitotecnia - DFT - ESALQ/USP. 


\section{INTRODUÇÃO}

As plantas daninhas possuem, geralmente, destacada habilidade na captação de recursos e adaptação ao ambiente. Dentre suas características que merecem destaque, incluem-se a elevada produção de diásporos e o seu potencial de disseminação. A viabilidade dos diásporos no solo por longo período de tempo resulta em problema para o controle (Masin et al., 2005). Dessa forma, a composição do banco de diásporos de plantas daninhas em solos cultivados e as especificidades de germinação de cada espécie têm se destacado como fatores de estudo indispensáveis para seu manejo, permitindo maior eficiência técnica, bem como a sustentabilidade dos agroecossistemas.

Entre os fatores do ambiente que mais influenciam a germinação de diásporos de diversas espécies estão o teor de água no solo, a presença ou ausência de luz e as amplitudes térmicas (Mennan, 2003; Penny \& Neal, 2003; Norsworthy $\&$ Oliveira, 2007). Muitas das variações desses fatores estão envolvidas na geração e regulação do banco de diásporos no solo, as quais têm permitido, por meio de estimativas e com o uso de modelos matemáticos, predizer as taxas de emergência de espécies ou servir na racionalização de herbicidas (Voll et al., 1997). Ao mesmo tempo, fatores ambientais - como a luz e a temperatura principalmente - podem afetar a dormência conforme a exigência de cada espécie, pois sabe-se que, quanto maior o nivel de dormência, maior será a especificidade dos fatores ambientais requeridos para sua superação (Benech-Arnold et al., 2000)

Nesse sentido, Otegui et al. (2005) avaliaram a influência da luz e da temperatura na taxa e velocidade de germinação de Paspalum guenoarum, sendo constatado que a temperatura alternada de $20 / 35{ }^{\circ} \mathrm{C}$ (dia/noite) foi a mais apropriada para expressar o potencial germinativo desta espécie, independentemente da luz. Já para a espécie Fatoua villosa, a luz estimulou a germinação, sendo constatadas diferenças maiores que $40 \%$ na taxa de germinação em relação ao tratamento na ausência de luz. Esses autores também verificaram que a espécie germina em ampla faixa de temperatura, de 15 até $40{ }^{\circ} \mathrm{C}$, sugerindo potencial de emergência dessa espécie em várias épocas do ano.

Quanto à espécie Alternanthera tenella, há ainda poucas informações sobre a necessidade térmica e luminosa para a sua germinação. Juntamente com Conyza bonariensis, essas espécies têm se destacado nos últimos anos como importantes plantas daninhas nas culturas de soja (Petter et al., 2007) e citros, as quais apresentaram elevado potencial competitivo. C. bonariensis, por exemplo, apresenta diversos problemas de manejo relacionados à resistência ao herbicida glyphosate (Moreira et al., 2007), sendo importante detalhar as exigências biológicas na sua germinação como futura estratégia de utilização como manejo. Já Digitaria ciliaris está entre as espécies de ocorrência mais comum na maioria das áreas agrícolas (Kobayashi \& Oyanagi, 2005). Segundo Kobayashi \& Oyanagi (2005), a germinação de $D$. ciliaris é estimulada pela flutuação diurna da temperatura e também pela mediação luminosa do fitocromo, embora o seu comportamento no banco de diásporos do solo seja ainda desconhecido. Atualmente, com o aumento do sistema de semeadura direta, é possível a redução da sua infestação nas lavouras (Voll et al., 1997), embora muito ainda precise ser estudado para o manejo adequado dessa planta daninha.

A compreensão das limitações na germinação e das características de cada espécie contribuem significativamente para as técnicas de controle e manejo a serem utilizadas. Assim, o percentual de emergência de plantas daninhas em pré-semeadura das culturas pode ser reduzido pelas práticas de manejo e, assim, suprimir as infestações e o banco de diásporos da espécie. Já a distribuição espacial das sementes no perfil do solo pode determinar a intensidade de emergência na fase inicial de desenvolvimento da cultura ou, ainda, simular práticas agrícolas (Gonzalez-Andujar, 1997). Portanto, com o intuito de aperfeiçoar o manejo das plantas daninhas e ampliar as informações das necessidades durante o seu processo germinativo, buscou-se avaliar a influência da luz e as variações da temperatura na taxa e velocidade de germinação das espécies Alternanthera tenella, Conyza bonariensis e Digitaria ciliaris. 


\section{MATERIAL E MÉTODOS}

O experimento foi realizado no Laboratório de Sementes do Departamento de Produção Vegetal da Escola Superior de Agricultura Luiz de Queiroz - ESALQ/USP. Inicialmente, as sementes de cada espécie foram coletadas em canteiros experimentais, pré-selecionadas e armazenadas em câmara fria a $7 \pm 1{ }^{\circ} \mathrm{C}$. Em seguida, as sementes passaram por soprador de ar, para permitir a sua homogeneização quanto a sua densidade e garantir a uniformidade amostral. As sementes de A. tenella foram destacadas dos seus frutos por fricção manual, para permitir maior contato com o papel de germinação e facilidade no seu manuseio. Posteriormente, as sementes foram dispostas em caixas gerbox previamente desinfetadas e com a base forrada com papel de germinação "germitest", umedecido com água destilada equivalente a 2,5 vezes a massa do papel seco. Utilizaram-se 50 sementes de cada espécie por caixa, as quais foram incubadas em germinadores verticais tipo BOD com controle de temperatura estabelecido conforme proposto.

Os tratamentos consistiram de variação de temperatura $(15 / 25,20 / 30,20 / 35$ e $30 /$ $40{ }^{\circ} \mathrm{C}$ ), considerando o período de 8 e 16 horas diurna/noturna, respectivamente, e ainda com e sem a presença de luz. As unidades experimentais (UE) foram compostas por cada caixa gerbox, sendo utilizadas quatro repetições por tratamento, totalizando $32 \mathrm{UE}$ para cada espécie de planta daninha (A. tenella, $C$. bonariensis e $D$. ciliaris). Para simular o escuro, foram utilizadas caixas gerbox pretas, tendo-se o cuidado para impedir o contato das sementes com a luz, inclusive durante a instalação e nas avaliações do experimento, as quais eram realizadas em sala fechada, somente com luz verde (Kendricks \& Frankland, 1983).

As avaliações constituíram-se de verificação da germinação diária em cada tratamento, após três dias da incubação, realizadas diariamente durante o período total de 40 dias do experimento. Foram consideradas como germinadas as sementes com protusão radicular igual ou superior a $1 \mathrm{~mm}$. Aos 27 dias após incubação, substituiu-se a tampa superior das caixas gerbox escuras por outra transparente, permitindo o fluxo luminoso da câmara de germinação para as sementes, com a intenção de confirmar o efeito da luz como promotor da germinação de cada espécie. A partir das avaliações de germinação diárias, calculou-se a taxa máxima (valor acumulado percentual) e o índice de velocidade de germinação (IVG), conforme Maguire (1962).

$O$ experimento foi realizado em esquema fatorial $4 \times 2$, em delineamento inteiramente casualizado, sendo constituído de quatro variações de temperatura e duas condições de luminosidade (com e sem a presença de luz), respectivamente. Os niveis de cada fator foram avaliados pelo teste DMS (Diferença Mínima Significativa), de acordo com a significância da interação, a 5\% de probabilidade, sendo ajustada regressão, quando necessária.

\section{RESULTADOS E DISCUSSÃO}

A interação entre temperatura e luz foi significativa $(\mathrm{p}<0,05)$ somente para a espécie C. bonariensis (Tabela 1). Para as demais espécies, as variáveis avaliadas demonstraram que os fatores agem isoladamente no processo de germinação, ao menos para as variações de temperatura e luz testadas. Conforme Zhou et al. (2005), algumas espécies precisam ser expostas por períodos prolongados à luz, mesmo que a temperatura esteja dentro de uma amplitude subótima para a germinação. Para outras, a breve exposição à luz é suficiente para desencadear o processo germinativo, embora necessitem de diversos ciclos de flutuação térmica para que esta ocorra. Logo, a interação entre luz e temperatura nem sempre é confirmada na germina ção das sementes de muitas espécies de plantas daninhas, conforme constatado para $A$. tenella e $D$. ciliaris

Quanto à espécie A. tenella, verificou-se que as flutuações térmicas testadas $(15 / 25$, $20 / 30,25 / 30$ e 30/40) pouco influenciaram o percentual, bem como o seu indice de velocidade de germinação, com médias obtidas variando de 78 a $96 \%$ e de 5,5 a 6,9 , respectivamente (Tabela 2). As temperaturas mais elevadas $\left(20 / 30,25 / 30\right.$ e $\left.30 / 40{ }^{\circ} \mathrm{C}\right)$ favoreceram a germinação dessa espécie, possivelmente pela sua alta adaptação a climas quentes e secos, conforme observado em condições naturais. A ausência de luz reduz a germinação 
Tabela 1 - Análise da variância dos efeitos da temperatura e da luz na germinação (G) e índice de velocidade de germinação (IVG) de sementes de Alternanthera tenella, Conyza bonariensis e Digitaria ciliaris

\begin{tabular}{|c|c|c|c|c|c|}
\hline \multirow{2}{*}{ Espécie } & \multirow{2}{*}{ Fonte de variação } & \multicolumn{2}{|c|}{ IVG } & \multicolumn{2}{|c|}{$\mathrm{G}(\%)$} \\
\hline & & $\mathrm{F}$ & CV $(\%)$ & $\mathrm{F}$ & CV (\%) \\
\hline \multirow{3}{*}{ Alternanthera tenella } & Temperatura & ns & \multirow{3}{*}{10,9} & 0,0473 & \multirow{3}{*}{10,1} \\
\hline & Luz & 0,0001 & & $<0,0001$ & \\
\hline & Temperatura x Luz & ns & & n.s & \\
\hline \multirow{3}{*}{ Digitaria ciliaris } & Temperatura & 0,0060 & \multirow{3}{*}{16,5} & $<0,0001$ & \multirow{3}{*}{15,5} \\
\hline & Luz & $<0,0001$ & & $<0,0001$ & \\
\hline & Temperatura x Luz & $\mathrm{ns}$ & & n.s & \\
\hline \multirow{3}{*}{ Conyza bonariensis } & Temperatura & $<0,0001$ & \multirow{3}{*}{17,5} & $<0,0001$ & \multirow{3}{*}{18,0} \\
\hline & Luz & $<0,0001$ & & $<0,0001$ & \\
\hline & Temperatura x Luz & $<0,0001$ & & $<0,0001$ & \\
\hline
\end{tabular}

ns $=$ não-significativo $(\alpha=0,05)$.

Tabela 2 - Germinação percentual e índice de velocidade de germinação de sementes de Alternanthera tenella e Digitaria ciliaris, em função da variação da luz e temperatura

\begin{tabular}{|c|c|c|c|c|c|c|}
\hline \multirow{3}{*}{ Espécie } & \multicolumn{6}{|c|}{ Germinação (\%) } \\
\hline & \multicolumn{4}{|c|}{ Temperatura } & \multicolumn{2}{|c|}{ Luz } \\
\hline & $15 / 25$ & $20 / 30$ & $25 / 30$ & $30 / 40$ & Presença & Ausência \\
\hline Alternanthera tenella & $81,5 \mathrm{ab}$ & $78,0 \mathrm{~b}$ & $82,5 \mathrm{ab}$ & $96,0 \mathrm{a}$ & $95,0 \mathrm{a}$ & $71,3 \mathrm{~b}$ \\
\hline \multirow[t]{4}{*}{ Digitaria ciliaris } & $43,5 \mathrm{~b}$ & $48,0 \mathrm{ab}$ & $49,8 \mathrm{a}$ & $39,8 \mathrm{~b}$ & $73,0 \mathrm{a}$ & $17,5 \mathrm{~b}$ \\
\hline & \multicolumn{6}{|c|}{ Índice de velocidade de germinação } \\
\hline & \multicolumn{4}{|c|}{ Temperaturas } & \multicolumn{2}{|c|}{ Luz } \\
\hline & $15 / 25$ & $20 / 30$ & $25 / 30$ & $30 / 40$ & Presença & Ausência \\
\hline Alternanthera tenella & $5,5 \mathrm{a}$ & $5,7 \mathrm{a}$ & $6,3 \mathrm{a}$ & $6,3 \mathrm{a}$ & $6,9 \mathrm{a}$ & $5,0 \mathrm{~b}$ \\
\hline Digitaria ciliaris & $5,7 \mathrm{~b}$ & $7,2 \mathrm{a}$ & $7,2 \mathrm{a}$ & $5,7 \mathrm{~b}$ & $10,4 \mathrm{a}$ & $2,5 \mathrm{~b}$ \\
\hline
\end{tabular}

* Médias seguidas por pelo menos uma mesma letra na linha não diferem significativamente entre si pelo teste DMS $(p<0,05)$.

e o IVG de A. tenella (Tabela 2). Para essa espécie, não se constatou nenhum dado comparativo até o momento na literatura nacional, em relação às exigências térmicas e de luminosidade durante a germinação, sendo necessários mais estudos que avaliem o efeito de temperaturas continuas e de intensidades luminosas na sua germinação.

Ao avaliar o comportamento de $D$. ciliaris, mesmo com baixos percentuais de germinação, verificou-se que os diásporos da espécie foram favorecidos com as variações térmicas de $20 / 30$ e $25 / 30{ }^{\circ} \mathrm{C}$, com distinta influência da luz na sua germinação e no IVG dessa espécie (Tabela 2). Verificou-se que o IVG também seguiu o mesmo padrão observado para a germinação, com os maiores valores constatados para as variações de temperatura de 20/30 e $25 / 30{ }^{\circ} \mathrm{C}$. Em estudos realizados por Kobayashi
$\&$ Oyanagi (2005), verificou-se que sementes de $D$. ciliaris demonstraram grande necessidade de luz para a sua germinação, mesmo quando presentes na superficie do solo. A promoção da germinação de sementes pela luz ocorre devido à conversão de fitocromos inativos para a forma ativa, por meio da luz vermelha (Thompson \& Grime, 1983). A forte dependência das sementes dessa espécie à luz, mesmo quando sujeitas às flutuações de temperatura avaliadas, sugere grande importância adaptativa, o que permite a sua germinação em ambientes abertos, como clareiras, ou mesmo nas entrelinhas ou bordas das culturas. De fato, isso pode ser observado em condições de campo, sugerindo modificações no manejo cultural para redução do banco de sementes de $D$. ciliaris.

Em relação a $C$. bonariensis, a interação dos fatores luz e temperatura indica maior 
especificidade ambiental para germinação de diásporos desta espécie daninha. Contudo, verificou-se grande amplitude de resposta à temperatura, com significativas restrições da espécie à ausência de luz. Conforme se observa na Tabela 3, as sementes de $C$. bonariensis apresentaram germinação satisfatória e similar para as variações de $15 / 25,20 / 30$ e 25/ $30{ }^{\circ} \mathrm{C}$, embora a elevação para $30 / 40{ }^{\circ} \mathrm{C}$ tenha inibido a sua ocorrência. Convém ressaltar que as sementes submetidas à temperatura de $30 / 40^{\circ} \mathrm{C}$ foram, no final do experimento, readaptadas ao germinador de temperatura de $25 / 30^{\circ} \mathrm{C}$, sendo constatada a sua germinação a partir do terceiro dia (dados não apresentados). Ao mesmo tempo, a ausência de luz inibiu significativamente $(p<0,05)$ a sua germinação e o IVG, podendo o efeito ser minimizado por temperaturas mais amenas, como pode ser constatado pela germinação de $67,0 \%$ a $15 /$ $25{ }^{\circ} \mathrm{C}$, mesmo na ausência de luz (Tabela 3). A observação de fotoblastia positiva para a espécie $C$. bonariensis já havia sido constatada por Vidal et al. (2007), embora os autores tenham verificado maiores percentuais de germinação com temperatura constante superior a $20{ }^{\circ} \mathrm{C}$. Para muitas espécies, como verificado para Xanthium strumarium, existe relação entre a luz e a amplitude térmica. Para esta espécie, foi constatado que temperaturas constantes diurnas e noturnas reduzem a necessidade de luz para a sua germinação. Por outro lado,

Tabela 3 - Germinação percentual e índice de velocidade de germinação de sementes de Conyza bonariensis, em função da variação da luz e temperatura

\begin{tabular}{|l|c|c|c|c|}
\hline \multirow{2}{*}{ Luz } & \multicolumn{4}{|c|}{ Germinação (\%) } \\
\cline { 2 - 5 } & \multicolumn{4}{|c|}{ Temperatura } \\
\cline { 2 - 5 } & $15 / 25$ & $20 / 30$ & $25 / 30$ & $30 / 40$ \\
\hline Presença & $82,5 \mathrm{aA}$ & $85,0 \mathrm{aA}$ & $85,5 \mathrm{aA}$ & $0,0 \mathrm{bA}$ \\
\hline Ausência & $67,0 \mathrm{aB}$ & $40,5 \mathrm{bB}$ & $3,0 \mathrm{cB}$ & $1,0 \mathrm{cA}$ \\
\hline & \multicolumn{5}{|c|}{ Índice de velocidade de germinação } \\
\cline { 2 - 5 } & \multicolumn{5}{|c|}{ Temperatura } \\
\hline & $15 / 25$ & $20 / 30$ & $25 / 30$ & $30 / 40$ \\
\hline Presença & $10,5 \mathrm{aA}$ & $11,9 \mathrm{aA}$ & $10,8 \mathrm{aA}$ & $0,0 \mathrm{bA}$ \\
\hline Ausência & $8,5 \mathrm{aB}$ & $5,1 \mathrm{bB}$ & $0,4 \mathrm{cB}$ & $0,2 \mathrm{cA}$ \\
\hline
\end{tabular}

* Médias seguidas por pelo menos uma mesma letra minúscula na linha e maiúscula na coluna não diferem entre si pelo teste DMS $(\mathrm{p}<0,05)$ quando as sementes são submetidas a ambientes com amplitude térmica variada, por exemplo, $25 / 30$ ou $20 / 30^{\circ} \mathrm{C}$, confirma-se a necessidade de elevada intensidade de luz, sendo condição indispensável para ocorrência da sua germinação (Norsworthy \& Oliveira, 2007). Nesse sentido, essas características adaptativas conferem maior distribuição temporal e espacial na germinação das diferentes espécies, permitindo sua sobrevivência em ambientes menos propícios ao seu desenvolvimento.

Para evidenciar o efeito da luminosidade no processo de germinação, ressalta-se o comportamento de A. tenella em temperatura de $30 / 40{ }^{\circ} \mathrm{C}$ durante os 40 dias de avaliação do experimento (Figura 1). Verificou-se que a luz aumentou a geminação dessa espécie, embora a sua ausência não se constitua em impedimento para a ocorrência. Aos 27 dias de avaliação, após a adição de luz, constatou-se leve aumento na germinação da espécie, atingindo valores muito próximos aos obtidos com o tratamento com luz aos 40 dias de avaliação.

Em relação a $D$. ciliaris $\left(20 / 35^{\circ} \mathrm{C}\right)$, verificou-se efeito pronunciado da luz no processo de germinação após adição desta aos 27 dias. $O$ percentual de germinação aumentou consideravelmente em cerca de $60 \%$, conferindo rápida resposta luminosa da espécie às variações do ambiente (Figura 2).

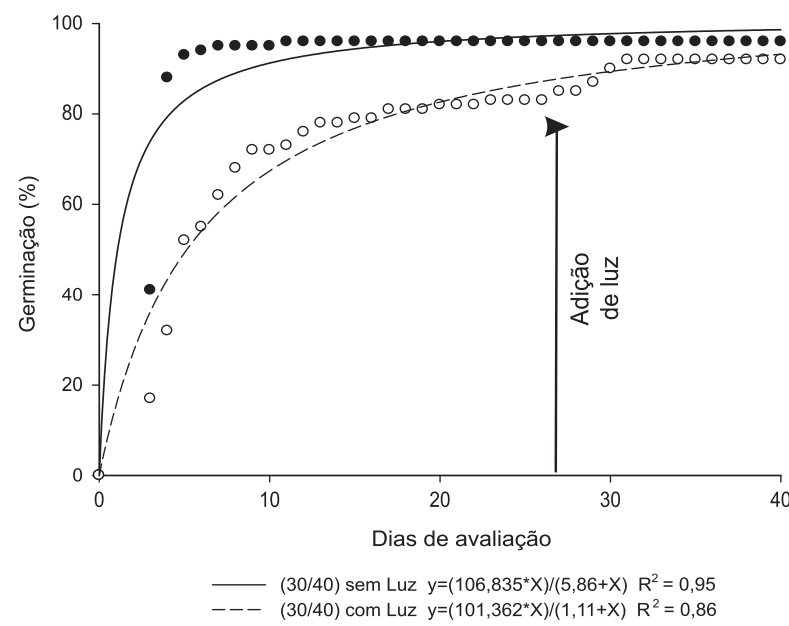

Figura 1 - Percentual de germinação de Alternanthera tenella sob condições de luz e escuro, para a temperatura de 30/ $40{ }^{\circ} \mathrm{C}$ noturna/diurna, respectivamente.

Planta Daninha, Viçosa-MG, v. 26, n. 3, p. 507-513, 2008 


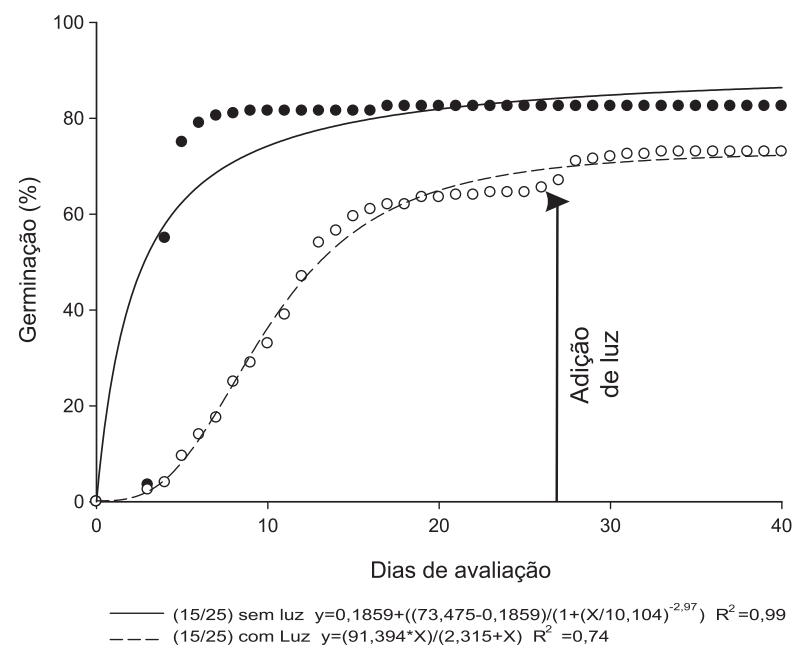

Figura 2 - Percentual de germinação de Digitaria ciliaris sob condições de luz e escuro, para a temperatura de $20 / 35^{\circ} \mathrm{C}$ noturna/diurna, respectivamente.

Para C. bonariensis, embora a espécie tenha apresentado resposta conjunta à temperatura e luminosidade, a presença da luz demonstrou efeito significativo $(p<0,05)$, sobretudo na velocidade de germinação (Tabela 2 e Figura 3). Para A. tenella e C. bonariensis, verificou-se que o efeito da luz foi principalmente na velocidade de germinação, e o percentual máximo para os tratamentos sem luz foi alcançado cerca de quatro e oito dias após o início da geminação com luz, respectivamente. Já para $D$. ciliaris, os efeitos ocorreram tanto no percentual de germinação como na sua velocidade, sendo constatada diferença de 16 dias entre os tratamentos com e sem luz em relação à máxima germinação aos 40 dias após instalação do experimento.

Conforme resultados observados, a presença de luz altera significativamente a germinação de todas as espécies, principalmente para D. ciliaris. Em relação à temperatura, as variações de $15 / 25,20 / 30,20 / 35$ e $30 / 40{ }^{\circ} \mathrm{C}$ afetam distintamente a germinação de A. tenella e $D$. ciliaris, sendo a maior delas (30/40) deletéria para C. bonariensis. As variações de luz e temperatura também foram significativas no indice de velocidade de germinação, com maior efeito constatado em $D$. ciliaris. Além disso, essa foi a única espécie a apresentar resposta da interação entre temperatura e luz no seu processo germinativo.

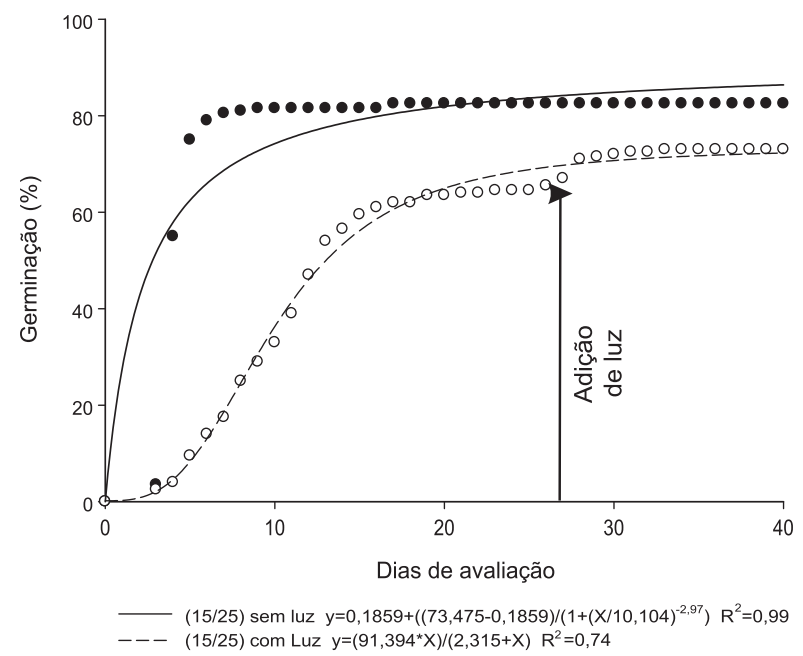

Figura 3 - Percentual de germinação de Conyza bonariensis sob condições de luz e escuro, para a temperatura de 15/ $25^{\circ} \mathrm{C}$ noturna/diurna, respectivamente.

Nesse sentido, espera-se que o conhecimento das características particulares de germinação dessas espécies possa se constituir em ferramenta para programas integrados de manejo delas. Ao mesmo tempo, a comprovação do estímulo luminoso na sua germinação pode despertar o interesse pelo controle cultural, assim como a técnica de utilização de resíduos culturais sobre o solo, para reduzir a emergência e posterior interferência dessas espécies nas áreas cultivadas.

\section{LITERATURA CITADA}

BENECH-ARNOLD, R. L. et al. Environmental control of dormancy in weed seed banks in soil. Field Crops Res., v. 67, n. 1, p. 105-122, 2000.

GONZALEZ-ANDUJAR, J. L. A matrix model for the population dynamics and vertical distribution of weed seedbanks. Ecol. Mod., v. 97, n. 1-2, p.117-120, 1997.

KENDRICKS, R. E.; FRANKLAND, B. Phytocrome and plant growth. In: ARNOLD, E. (Ed.). Southampton: The Camelot Press, 1983. 76 p.

KOBAYASHI, H.; OYANAGI, A. Digitaria ciliaris seed banks in untilled and tilled soybean fields. Weed Biol. Manag., v. 5, n. 1, p. 53-61, 2005.

MAGUIRE, J. D. Speed of germination-aid in selection and evaluation for seedling emergence and vigor. Crop Sci., v. 2, n. 2 , p. 176-177, 1962 
MASIN, R. et al. Weed turf: A predictive model to aid control of annual weeds in turf. Weed Sci., v. 53, p. 193201, 2005 .

MENNAN, H. The effects of depth and duration of burial on seasonal germination, dormancy and viability of Galium aparine and Bifora radians seeds. J. Agron. Crop Sci., v. 189 , p. 304-309, 2003

MOREIRA, M. S. et al. Resistência de Conyza canadensis e C. bonariensis ao herbicida glyphosate. Planta Daninha, v. 25, n. 1, p. 157-164, 2007.

NORSWORTHY, J. K.; OLIVEIRA, J. Light and temperature requirements for common cocklebur (Xanthium strumarium) germination during after-ripening under field conditions. Weed Sci., v. 55, p. 227-234, 2007.

OTEGUI, M. B.; PÉREZ, M. A.; SOUZA MAIA, M. Efecto de la temperatura y la luz en la germinación de semillas de Paspalum guenoarum. R. Bras. Sementes, v. 27, n. 1, p. 190-194, 2005.
PENNY, G. M.; NEAL, J. C. Light, temperature, seed burial, and mulch effects on mulberry weed (Fatoua villosa) seed germination. Weed Technol., v. 17, p. 213-218, 2003

PETTER, F. A. et al. Associações entre o herbicida glyphosate e inseticidas na cultura da soja roundup ready. Planta Daninha, v. 25, n. 2, p. 389-398, 2007.

THOMPSON, K.; GRIME, J. P. A comparative study of germination in response to diurnally-fluctuating temperatures. J. Appl. Ecol., v. 20, n. 1, p. 141-156, 1983.

VIDAL, R. A. et al. Impacto da temperatura, irradiância e profundidade das sementes na emergência e germinação de Conyza bonariensis e Conyza canadensis resistentes ao glyphosate. Planta Daninha, v. 25, n. 2, p. 309-315, 2007.

VOLL, E.; KARAM, D.; GAZZIERO, D. L. P. Dinâmica de populações de capim-colchão (Digitaria ciliaris) sob manejos de solo e de herbicidas. Pesq. Agropec. Bras., v. 32, n. 4, p. 373-378, 1997. 